\title{
Differences in Serum Steroid Hormones Concentrations in Spontaneously Hypertensive Rats (SHR) - an Animal Model of Attention-Deficit/Hyperactivity Disorder (ADHD)
}

\author{
A. KOZLOWSKA ${ }^{1}$, P. WOJTACHA ${ }^{2}$, M. RÓWNIAK ${ }^{3}$, M. KOLENKIEWICZ ${ }^{4}$, M.-L. TSAI ${ }^{5}$
}

${ }^{1}$ Department of Human Physiology, School Medicine, Collegium Medicum, University of Warmia and Mazury, Olsztyn, Poland, ${ }^{2}$ Department of Industrial and Food Microbiology, Faculty of Food Science, University of Warmia and Mazury, Olsztyn, Poland, ${ }^{3}$ Department of Animal Anatomy and Physiology, Faculty of Biology and Biotechnology, University of Warmia and Mazury, Olsztyn, Poland, ${ }^{4}$ Department of Pathophysiology, School Medicine, Collegium Medicum, University of Warmia and Mazury, Olsztyn, Poland, ${ }^{5}$ Department of Biomechatronic Engineering, National Ilan University, Yilan, Yilan City, Taiwan

Received March 14, 2018

Accepted July 10, 2018

Epub Ahead of Print October 23, 2018

\section{Summary}

Spontaneously hypertensive rats are the most common animal model used to study attention deficit hyperactivity disorder (ADHD). The present study investigated the levels of steroid hormones in the bloodstream of hypertensive rats and its normotensive control strain, Wistar-Kyoto rats, to check if there are any hormonal differences between both strains at the onset of ADHD. Plasma samples were collected from young (5-weekold) and mature (10-week-old) male hypertensive and normotensive rats to determine the serum level of testosterone, $17 \beta$-estradiol, free estriol, progesterone, corticosterone and cortisol using ELISA kits. The results showed statistically significant increases in serum levels of testosterone and free estriol in 10-week-old hypertensive and normotensive rats when compared to 5-week-old animals. Moreover, the concentrations of progesterone, corticosterone and cortisol were significantly elevated in 10-week-old hypertensive rats when compared to 5 -week-old animals of both strains as well as 10-week-old normotensive rats. Hormonal differences observed between 10-week-old hypertensive and normotensive rats were also accompanied by differences in the volumes of lateral ventricles as well as the third ventricle and cerebral aqueduct. In conclusion, elevated contents of progesterone, corticosterone and cortisol in hypertensive rats may be associated not only with ADHD but also with developing hypertension. This question needs further study.

\section{Key words}

Hormones • ADHD • Spontaneously hypertensive rats

\section{Corresponding author}

A. Kozłowska, Department of Human Physiology, School Medicine, Collegium Medicum, University of Warmia and Mazury in Olsztyn, Warszawska Av, 30, 10-082 Olsztyn, Poland. E-mail: kozlowska.anna@uwm.edu.pl

\section{Introduction}

It is estimated that attention-deficit/hyperactivity disorder (ADHD) affects $10 \%$ of boys and $5 \%$ of girls at elementary school age (Dulcan 1997). This disorder is a developmental condition of inattention and distractibility, with or without associating hyperactivity and it is also characterized by susceptibility to distraction (Nagui 2009). It has been reported that anatomical abnormalities in the brain could be attributable to symptoms of ADHD (Hsu et al. 2010). Neuroimaging studies of children's brains with ADHD have shown that the main putative brain regions involved in this condition are the prefrontal cortex (Zang et al. 2007), striatum (Scheres et al. 2007) and cerebellum (Castellanos et al. 1996). However, abnormalities have also been found in other brain regions, such as the anterior cingulate cortex 
(Zang et al. 2007) and substantia nigra (Romanos et al. 2010).

There is also data indicating that steroid hormones may play a role in the pathogenesis of ADHD. It is not surprising as they are engaged in the brain organization, plasticity and modulation of neurotransmitter system (McEwen 1992, Morris et al. 2004) and there is a sex bias in ADHD (Gaub and Carlson 1997). For example, it is suggested that higher prenatal testosterone $(\mathrm{T})$ exposure is associated with a greater risk of developing disruptive behavior disorders. This suggestion is partly supported by King et al. (2010) who found that the exposure of spontaneously hypertensive rats (SHRs; an animal model of ADHD) to elevated T-levels during early development resulted in additional deficits in spatial memory. In addition, various neurocognitive effects of $\mathrm{T}$ on boys and girls with ADHD were observed and they were sex-specific (Wang et al. 2017). Finally, medical drugs such as methylphenidate, which is widely used to treat ADHD (Burcu et al. 2016), can potentially diminish T-levels and, in consequence, delay puberty onset (Ramasamy et al. 2014). Estrogens and progesterone $\left(\mathrm{P}_{4}\right)$ have also been proposed to play an important role in ADHD, because they are synthesized de novo in the cerebellum during critical developmental periods in rats (Dean and McCarthy 2008). In addition, a low level of estrogens in women with ADHD is associated with intensity of symptoms (Roberts 2016). Furthermore, estrogens can increase visual and place memory in rats (Luine et al. 2003) as well as attention in macaques (Shively and Bethea 2004), and both memory and attention are deficient in children with ADHD (Holmes et al. 2014). Finally, studies in gonadectomized male mice treated with 1-methyl-4-phenyl-1,2,3,6tetrahydropyridine or with methamphetamine-induced neurotoxicity have shown that estrogens are engaged in the neuroprotection of the nigrostriatal dopaminergic system (Dluzen 2000), which is dysfunctional in children with ADHD (Romanos et al. 2010). The participation of corticosteroids in the course of ADHD should also be considered since the level of cortisol (CT) was lowered in children with ADHD (Isaksson 2014). A similar observation was reported in SHR rats with regard to corticosterone (CTT) (King et al. 2010). It is worth mentioning that low levels of corticosteroids may indicate abnormalities in the activity of the hypothalamicpituitary-adrenal (HPA) axis, which is involved in emotion, learning and attention (Smith 2006).

All of these findings suggest that serum concentrations of various steroid hormones should be altered in ADHD subjects. To verify this hypothesis, these concentrations were evaluated in SHRs, which are the best-validated animal model of ADHD (Sagvolden and Johansen 2012) with Wistar-Kyoto (WKY) rats serving as a control. The following hormones were tested: T, 17 $\beta$-estradiol $\left(\mathrm{E}_{2}\right)$, free estriol $\left(\mathrm{E}_{3}\right), \mathrm{P}_{4}, \mathrm{CTT}$ and CT. Considering that in SHR rats the anatomical abnormalities in the brain associated with ADHD are observed in the juvenile animals (5-week-old) and they disappear in mature animals (10-week-old) (Hsu et al. 2010), the highest alterations in hormone serum levels due to ADHD should be expected before puberty (5-week-old animals). As mature SHRs are also one of the most common animal models of hypertension in humans (Louis and Howes 1990), hormonal changes after puberty (10-week-old animals) maybe associated with hypertension. Since ADHD is more common in boys than in girls (Gaub and Carlson 1997), male SHRs were chosen.

\section{Methods}

\section{Animals}

A total of twenty-four male rats were used in the present study. All of the animals were divided into four groups: (1) 5-week-old SHR rats $(\mathrm{n}=6)$; (2) 5-week-old WKY rats $(n=6)$; (3) 10-week-old SHR rats $(n=6)$, and (4) 10-week-old WKY rats $(n=6)$. The mean $( \pm \mathrm{SD})$ weight of animals in the individual groups was: (1) $120.08 \pm 6.30$; (2) $115.08 \pm 4.65$; (3) $269.58 \pm 8.48$; and (4) 254.94 \pm 7.91. All experiments were carried out in accordance with the European Union Directive for animal experiments (2010/63/EU) and were approved by the Local Ethical Commission of the University of Warmia and Mazury in Olsztyn (no. 43/2014). The 3-week-old SHR and WKY rats were obtained from Charles River (Germany) and were transported to the animal house at the Institute of Animal Reproduction and Food Research of the Polish Academy of Sciences (Olsztyn, Poland) where they were housed in pairs or threes to prevent isolation stress. The temperature-controlled $\left(21 \pm 1^{\circ} \mathrm{C}\right)$ and ventilated (12-20 exchanges/h) animal room was maintained on a 12/12 h light/dark cycle (lights on from 6 a.m. to 6 p.m.). All animals were fed with a grain mixture (VRF1 diet; Charles River, Germany) and tap water ad libitum. All efforts were made to minimize animal suffering and to use the minimum number of animals necessary to produce reliable scientific data. 


\section{Tissue preparation}

Rats were deeply anesthetized with an intraperitoneal injection of pentobarbital (Biowet, Poland; $50 \mathrm{mg} / \mathrm{kg}$ ), then, the abdomen was opened and blood was drawn from the vena cava into EDTA tubes (42110, FLMEDICAL, Poland) (Palombo et al. 2000). Blood samples were collected from all animals between 7 a.m. and 8 a.m. In each animal blood was taken within time shorter than $3 \mathrm{~min}$ to avoid the initiation of the pituitary stress response (Vahl et al. 2005). After collection of blood samples, all animals were transcardially perfused with saline $(0.9 \%)$ followed by $4 \%$ paraformaldehyde (pH 7.4; 1040051000, Merck, Germany) in phosphate-buffered saline (PBS; P5493, Sigma Aldrich, Germany).

Following perfusion, the brains were carefully dissected out from the skulls and post-fixed by immersion in the same fixative for $24 \mathrm{~h}$, washed three times in $0.1 \mathrm{M}$ phosphate buffer $\left(\mathrm{pH}=7.4,4{ }^{\circ} \mathrm{C}\right)$ and then stored for 3-5 days in graded solutions $(10 \%, 20 \%$ and $30 \%)$ of sucrose (363-117720907, ALCHEM, Poland) in 1xPBS at $4{ }^{\circ} \mathrm{C}$ until they sunk. Finally, the brains were frozen and then coronally sectioned at a thickness of $10 \mu \mathrm{m}$ using a cryostat (HM525 Zeiss, Germany). The sections were stored at $-80^{\circ} \mathrm{C}$ until further processing.

Immunoenzymatic determination (ELISA) of steroid hormone concentrations in rat serum

The measurements of steroid hormones: testosterone, estradiol, free estriol, progesterone, corticosterone and cortisol in rat plasma were done with an ELISA test according to the manufacturer's instructions. All ELISA tests were produced by DRG Instruments (Germany; Table 1). The absorbance in ELISA test plate was measured by plate reader TECAN infinite M200 PRO (Austria) at the wavelength $\lambda=492 \mathrm{~nm}$.

Table 1. List of ELISA kits used for the determination of steroid hormones concentrations in rat serum.

\begin{tabular}{|c|c|c|c|}
\hline ELISA kit & Catalog number and manufacturer & $\begin{array}{c}\text { Intra Assay } \\
\text { Variation CV [\%] }\end{array}$ & $\begin{array}{c}\text { Inter Assay } \\
\text { Variation CV [\%] }\end{array}$ \\
\hline Testosterone ELISA & $\begin{array}{l}\text { EIA-1559. DRG Instruments } \\
\text { (Afify et al. 2010) }\end{array}$ & $3.593 \%$ & $7.126 \%$ \\
\hline Estradiol ELISA & $\begin{array}{l}\text { EIA-2693. DRG Instruments } \\
\text { (Chistyakov et al. 2010) }\end{array}$ & $8.970 \%$ & $10.870 \%$ \\
\hline Free Estriol ELISA & $\begin{array}{l}\text { EIA-1612. DRG Instruments } \\
\text { (Klocke et al. 2014) }\end{array}$ & $3.930 \%$ & $7.530 \%$ \\
\hline Progesterone ELISA & $\begin{array}{l}\text { EIA-1561. DRG Instruments } \\
\text { (Inegbenebor et al. 2009) }\end{array}$ & $6.416 \%$ & $6.630 \%$ \\
\hline Corticosterone ELISA & $\begin{array}{l}\text { EIA-4164. DRG Instruments } \\
\quad \text { (Kazemi et al. } 2011)\end{array}$ & $3.096 \%$ & $6.010 \%$ \\
\hline Cortisol ELISA & $\begin{array}{l}\text { EIA-1887. DRG Instruments } \\
\quad \text { (Kalshetti et al. 2015) }\end{array}$ & $5.630 \%$ & $6.930 \%$ \\
\hline
\end{tabular}

\section{Immunohistochemistry}

Brain sections were processed for DAB immunohistochemistry using primary antisera and species-specific secondary antibodies. All staining procedures were carried out in humid chambers (Immuno Slide Staining Trays, R64001-E, Pyramid Innovation Ltd., UK) and at room temperature.

\section{DAB immunohistochemistry}

The sections designated for morphometric and stereological procedures (every $25^{\text {th }}$ section in the single brain) were processed for a routine immunoperoxidase labeling using DAB as a chromogen (Dako Liquid DAB + Substrate Chromogen System, K3468, Denmark). After triple-washing cold PBS, the sections were pre-incubated for $30 \mathrm{~min}$ in $0.3 \% \mathrm{H}_{2} \mathrm{O}_{2}$ diluted in methanol and then for 60 min with a solution of $10 \%$ normal horse serum (diluted in PBS). The sections were then incubated overnight with a solution of primary antibodies directed towards neuron-specific nuclear protein $\mathrm{NeuN}$ (pan-neuronal marker; Anti-NeuN Antibody, clone A60, MAB377; Merck Millipore, Poland; working dilution 
1:1,000). The antibodies were diluted in PBS containing Triton X-100 (0.3-0.5\%) and $1 \%$ normal horse serum. In the next step, after triple-washing in cold PBS, the sections were incubated for $60 \mathrm{~min}$ with ImmPRESS Reagent, washed in cold PBS and incubated with a 3.3-diaminobenzidine substrate-chromogen solution (ImmPRESSTM UNIVERSAL REAGENT AntiMouse/Rabbit IgG PEROXIDASE, MP-7500; Vector Laboratories, Inc.; Burlingame, CA, USA). Finally, the sections were rinsed in tap water, dehydrated through graded alcohol series (POCH, Poland), cleaned in xylene and mounted in DPX (DPX Mountain for histology; 44581, Sigma Aldrich, Germany).

\section{Controls}

The antibody against neuron-specific nuclear protein NeuN used in the present study is an excellent marker for neurons in the central and peripheral nervous systems (Mullen et al. 1992). To test the secondary antibody specificity, the omission and replacement of all primary antisera by non-immune sera or PBS was applied. No observable immunoreactions had proven specificity.

\section{Stereological analyses}

Volumetric measurements were done using image-analysis software Fiji (Madison, USA). The following structures were taken into consideration in the WKY and SHR rats at each matched age: lateral ventricles (left and right) and third ventricle together with cerebral aqueduct. Measurements were done on evenly spaced sections arranged from the rostral to the caudal extent of the brain. Every $25^{\text {th }}$ section was stained using DAB method and antibody against NeuN protein from the level where the prefrontal cortex arrived to the end of the cerebellum. All these sections were then digitalized with magnification $5.0 \mathrm{x}$ using PathScan Enabler IV Histology Slide Scanner (Praha, Czech Republic). On each digital slice from the bregma 2.52 (Paxinos and Watson 2005) the boundaries of the individual brain ventricles (right and left lateral ventricles as well as $3^{\text {rd }}$ ventricle in conjunction with cerebral aqueduct) were outlined by a mouse-driven cursor. The number of sections analyzed per specific ventricle depended on the brain size and these numbers were as follows: right and left lateral ventricles: $25-28$ and $3^{\text {rd }}$ ventricle with conjunction of cerebral aqueduct: 29-32. Lengths differences were mostly due to the natural variability among subjects as well as strain and age volumetric differences. The total volumes of the individual brain ventricles were calculated according to the formula proposed by DeVito et al. (1989), in which the total volume of a structure (Vo) is the sum of the subvolumes of the sections through the structure $(\mathrm{Vn})$. The outlined areas depicting boundaries of the individual brain ventricles on the studied sections with the thickness $250 \mu \mathrm{m}$ (space between sections) were subvolumes.

\section{Preparation of images}

In the first step, all NeuN stained sections were digitalized using PathScan Enabler IV Histology Slide Scanner (Praha, Czech Republic) receiving images with a quality of $5.0 \mathrm{x}$ objective. These digital images were slightly modified to optimize the image resolution, brightness and contrast using CS4, version 11.0, software (Adobe Systems Inc., San Jose, CA, USA).

\section{Statistical analysis}

The statistical differences between groups of data (WKY vs. SHR at each matched age) were analyzed by one-way ANOVA followed by a Tukey test $(* \mathrm{P} \leq 0.05$, ** $\mathrm{P} \leq 0.01$ and $* * * \mathrm{P} \leq 0.001)$ using GraphPad Prism 6 software (GraphPad Software, La Jolla, CA, USA).

\section{Results}

The serum steroid hormone concentrations in the SHR and WKY rats

A significant increase of the serum content of $\mathrm{T}$ (Fig. 1A) and $\mathrm{E}_{3}$ (Fig. 1C) was noted in the 10-weekold SHR and WKY rats when compared to the juvenile animals. However, in both age groups, the $\mathrm{T}$ contents did not differ between SHR and WKY rats (Fig. 1A). Moreover, the concentrations of $\mathrm{P}_{4}$ (Fig. 1D), CCT (Fig. 1E) and CT (Fig. 1F) were significantly elevated in 10-week-old SHR rats when compared to 5-week SHR and 10 -week-old WKY rats (Figs 1D-F). No statistically significant differences in the serum levels of $E_{2}$ were found between age groups or between the strains at any of the ages studied (Fig. 1B).

The volumetric measurements of the brain ventricular system in the SHR and WKY rats

The brain ventricular system is enlarged in SHR rats when compared to that in the WKY rats (Figs 2-3). The volumes of the lateral ventricles in 5-week-old SHR rats (Figs $2 \mathrm{~A}$ and 3B) are approximately one-third larger than in WKY rats (Figs $2 \mathrm{~A}$ and 3A). The volumetric difference increases with age and in 10-week- 
old SHR rats these ventricles are two fold larger (Figs 2A and 3D) than in the WKY rats (Figs $2 \mathrm{~A}$ and $3 \mathrm{C}$ ). The total volumes composed of the third ventricles and cerebral aqueduct in the 5-week-old SHR were significantly greater than those of WKY rats (Fig. 2B). Moreover they also are one-quarter larger in the 10-weekold SHR rats compared to the 10-week-old WKY rats (Fig. 2B).
A

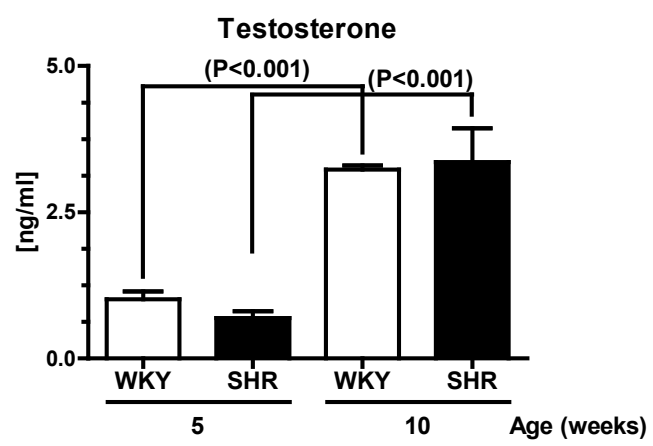

C

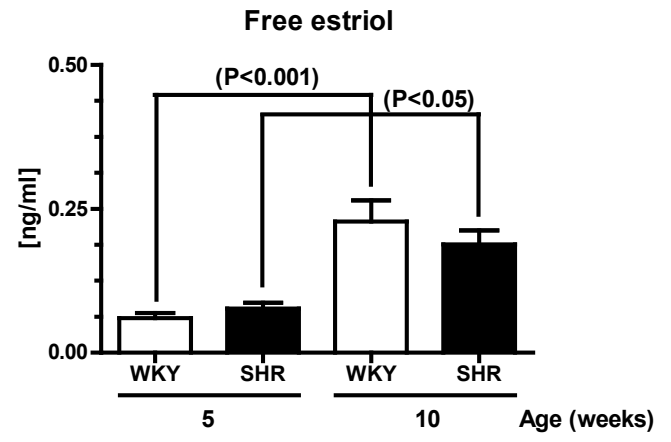

E

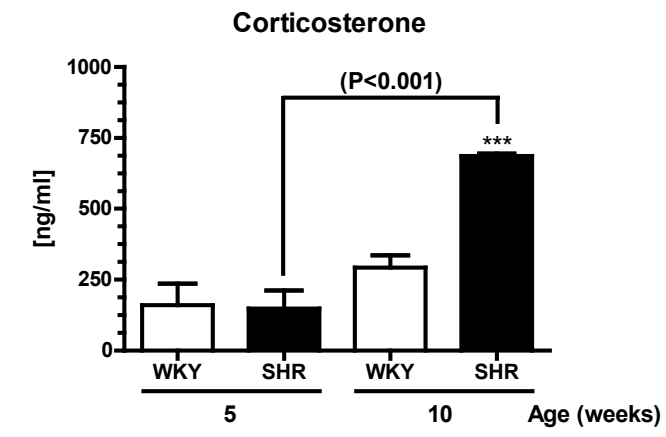

B

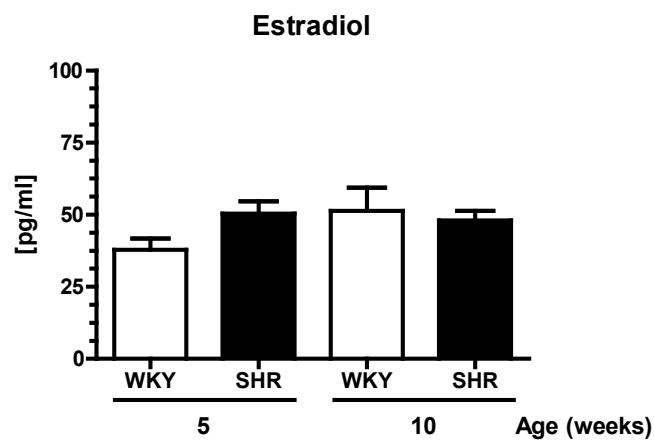

D

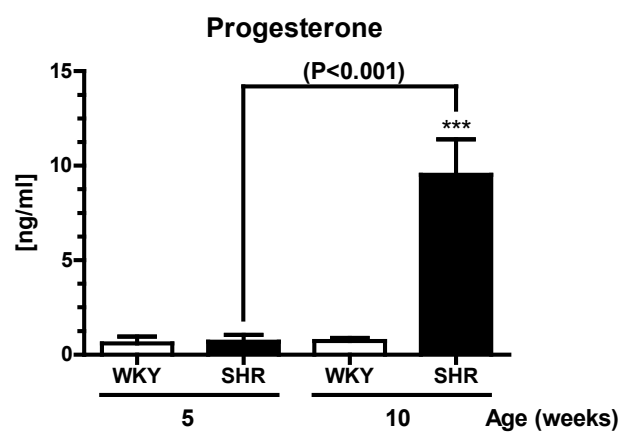

F

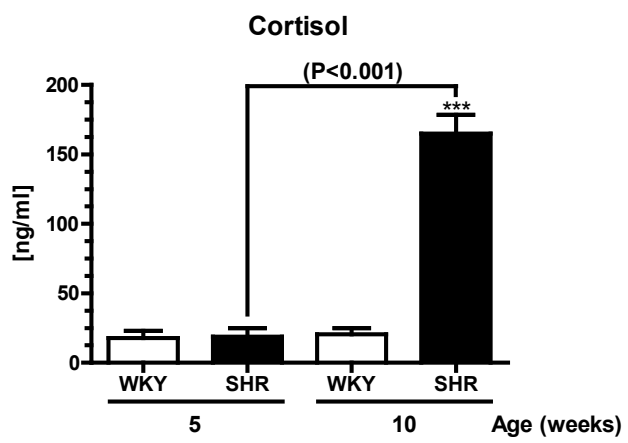

Fig. 1. Mean ( \pm SEM) concentrations of serum testosterone $(\mathbf{A})$, estradiol $(\mathbf{B})$, free estriol $(\mathbf{C})$, progesterone $(\mathbf{D})$, corticosterone $(\mathbf{E})$ and cortisol (F) in the 5 and 10 weeks old spontaneously hypertensive rat (SHR, $n=6$ in each group) and Wistar-Kyoto rat (WKY, $n=6$ in each group). $* * *-$ indicate differences $(\mathrm{P}<0.001)$ between the WKY and SHR rats. $\mathrm{P}<0.05 ; \mathrm{P}<0.001-$ indicates differences between the WKY and SHR before and after puberty. 
A

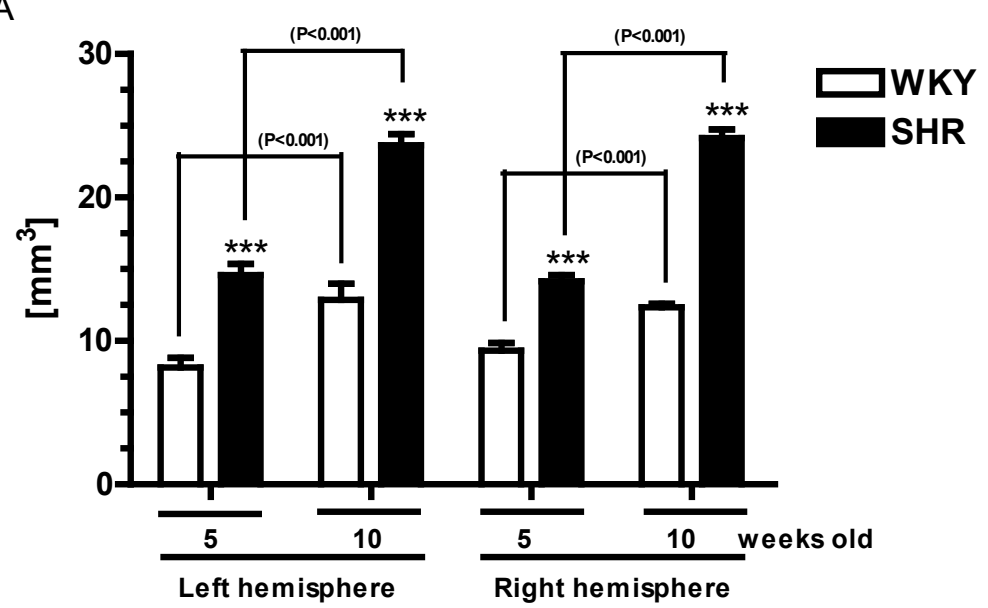

B

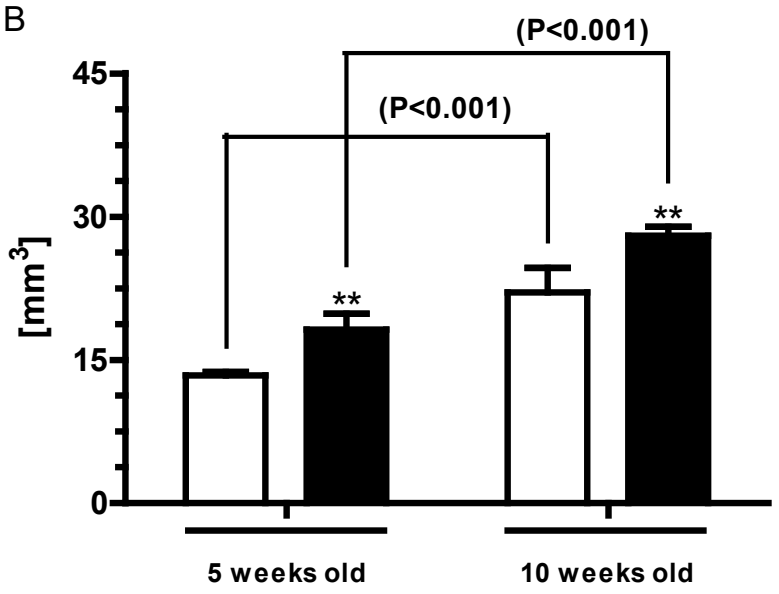

WHR
5 SHR

B

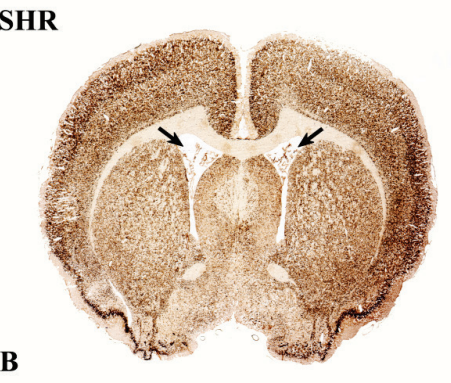

10 SHR

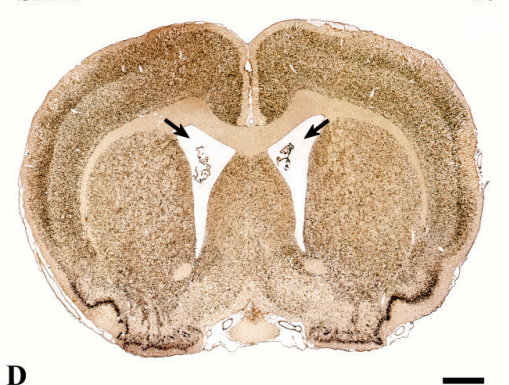

5

10
Fig. 2. The volumetric measurements of the brain ventricular system in the 5 and 10 weeks old spontaneously hypertensive rat (SHR, $n=6$ in each group) and Wistar-Kyoto rat (WKY, $\mathrm{n}=6$ in each group) rats. (A) lateral ventricles, (B) third ventricles and cerebral aqueduct. Data were expressed as mean standard deviation (SD). $* * ; * * *$ - indicate differences $(\mathrm{P}<0.01 ; \mathrm{P}<0.001)$ between the WKY and SHR rats. $\mathrm{P}<0.001$ - indicates differences between the WKY and SHR before and after puberty.

Fig. 3. Low-magnification photomicrographs of coronal sections through the brain of the WKY (A and $\mathbf{C}$ ) and SHR (B and D) rats illustrating enlargement of the left and right lateral ventricles (arrows) in SHR rats. Note that the size differences are bigger in 10-weeks old animals ( $C$ and $\mathbf{D}$ ) when compared to the 5-weeks old animals (A and B). Scale bar: $1 \mathrm{~mm}$.

\section{WKY}

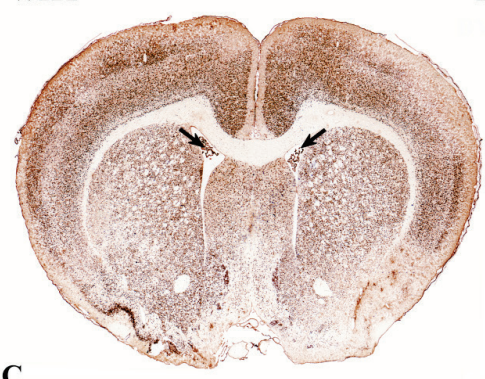

C

D 


\section{Discussion}

The present experiment showed that the serum steroid hormone contents differed significantly between the SHR and WKY rats; however, these differences were only evident in 10-week-old animals. The most striking differences were observed in $\mathrm{P}_{4}, \mathrm{CCT}$ and $\mathrm{CT}$ content, with subtle differences in the $\mathrm{T}$ and $\mathrm{E}_{3}$ content. Moreover, the differences in serum steroid hormones levels in the 10-week-old SHR rats were accompanied by twofold greater volumes of the brain lateral ventricles in those animals when compared to the WKY rats. An enlarged ventricular system was previously reported in mature SHR rats (Bendel and Eilam 1992). Salerno et al. (1992) suggested that standing hypertension results in structural changes in the human brain, e.g. a rise in mean volumes of the right and left lateral ventricles.

\section{Testosterone}

The immunoenzymatic determination revealed a significant increase of the serum T-content in the 10-week-old SHR and WKY rats when compared to the juvenile animals. However, in both age groups, the T-contents did not differ between SHR and WKY rats. The first phenomenon, i.e. a significant increase in the serum $\mathrm{T}$-content in the 10 -week-old animals is not surprising and quite easy to explain. It is well known that the testosterone level in rats is low in the prepubertal period, but it increases significantly during puberty (Döhler and Wuttke 1975). 5-week-old SHR and WKY rats are prepubertal, while 10-week-old animals are postpubertal. The second phenomenon, i.e. the lack of differences in T-contents between SHR and WKY rats in both age groups, is more difficult to explain and very intriguing. There is consensus that 5-6-week-old SHR rats are in the course of ADHD while with age they develop hypertension (Reckelhoff et al. 1998). However, it seems that both of these syndromes have no or very little impact on the serum T-content according to present results. There are many studies in rats (Wall et al. 1992, Dorn et al. 2009) as well as a few in humans (Wang et al. 2017, Pompa et al. 2007, Yu and Shi 2009) which try to explain the exact role of $\mathrm{T}$ in the course of ADHD. However, these results are sometimes contradictory. For example, there is a hypothesis that prenatal $\mathrm{T}$-exposure increases the risk of ADHD occurrence in boys (Martel and Roberts 2014). In support, some authors have revealed a significant positive relationship between T-concentration in saliva and aggressive behavior in adolescents (Pompa et al. 2007, Yu and Shi 2009). However, other authors did not find such a relationship (Dorn et al. 2009, Wang et al. 2017). There is also evidence that the serum T-levels were significantly higher in castrated juvenile and T-treated SHR rats than in WKY rats (Pompa et al. 2007). On the other hand, the salivary levels of this hormone in children with ADHD (boys and girls) did not change significantly in the group treated with methylphenidate or the untreated group (the intact group was not tested; Wang et al. 2017). It should be noted, however, that there was early androgen treatment in males (King et al. 2010). This fact coincides well with brain abnormalities and symptoms observed in SHR rats and ADHD patients (Castellanos et al. 1996, Castellanos et al. 2002, Sontag et al. 2011). The role of $\mathrm{T}$ in hypertension is also strongly postulated (Louis and Howes 1990, Yu and Shi 2009). For example, the serum T-level in the SHR male rats is high in the 12-week-old animals and is accompanied by high blood pressure (Reckelhoff et al. 1998). This observation corresponds with the results of Huisman et al. (2006), who reported that the serum $\mathrm{T}$ content was significantly higher in hypertensive humans of both sexes when compared to the normotensive controls. The lack of differences between 10-week-old SHR and WKY rats in the T-contents observed in the present study may be due to the fact that hypertension develops in 12-week-old SHR rats and, at that time, the T-level is much higher (Reckelhoff et al. 1998).

\section{Estrogens}

The results demonstrated that the serum levels of $E_{2}$ and $E_{3}$ do not differ in SHR and WKY rats. $E_{2}$ contents also do not differ in 5- and 10-week-old animals, but $\mathrm{E}_{3}$ is significantly increased in 10-week-SHR and WKY rats. The roles of $\mathrm{E}_{2}$ and/or $\mathrm{E}_{3}$ in $\mathrm{ADHD}$ are poorly documented, although existing data suggest neuroprotective actions of both of these hormones (Sherwin 2002, Xiao and Becker 1994, Reaven and Chang 1992). For example, $E_{2}$ positively impacts some aspects of cognitive function (Sherwin 2002). Moreover, $\mathrm{E}_{2}$ similarly to $\mathrm{P}_{4}$, can lead to an increase in the dopamine level in the striatum of female rats, however, unfortunately this phenomenon was not observed in male rats (Xiao and Becker 1994). $E_{3}$ is considered to regulate blood glucose concentration (Yamabe et al. 2014) and, in this way, relieve symptoms of ADHD in SHR rats, as it has been previously proposed for $\mathrm{P}_{4}$ and CT (Reaven and Chang 1992, Ryan and Enns 1988). The roles of 
$E_{2}$ and/or $E_{3}$ in hypertension are rather limited as the blood pressure in the SHR rats is independent of estrogen (Reckelhoff et al. 1998).

\section{Progesterone}

The results demonstrated that the serum levels of $\mathrm{P}_{4}$ do not differ in 5-week-old SHR and WKY rats or in 10-week-old WKY rats, but in 10-week-old SHR rats the level of this hormone is highly elevated. Such results would suggest rather the role of $\mathrm{P}_{4}$ in development of hypertension, but some roles of this hormone in ADHD are also postulated. For example, it was reported that ADHD symptoms were significantly reduced in children treated with high doses of $\mathrm{P}_{4}$ (Nadjafi 2010, Schilling 2014). The positive role of $P_{4}$ in ADHD may be supported by the results of Hsu et al. (2010) who found significant decrease in the striatal volume in the juvenile SHR rats (5-week-old) which was not observed in postpubertal animals (8-10-weeks-old). Similarly, significant differences in the caudate volume existing between ADHD children and healthy controls diminished with age studied (Castellanos et al. 2002). There is also a suggestion that $\mathrm{P}_{4}$, together with $\mathrm{CT}$, may relieve ADHD symptoms in another manner, namely by modulation of insulin resistance and, in this way, regulate glucose levels. This suggestion is supported by studies showing that both of these steroids lead to decreased maximum insulin binding and $\left[{ }^{14} \mathrm{C}\right] 3-\mathrm{O}$-methylglucose transport in cultured female virgin rat adipocytes (Ryan and Enns 1988). A similar effect was also observed in the juvenile and mature SHR rats where maximal insulinstimulated glucose transport by isolated adipocytes was lower than in WKY rats (Reaven and Chang 1992). The role of $\mathrm{P}_{4}$ in hypertension should also not be excluded. For example, it was reported that elevated levels of this hormone can exert antihypertensive effects in rats (Wambach and Higgins 1979). Interestingly, elevated $P_{4}$ content accompanied by enlargement of brain ventricles in the 10-week-old SHR rats observed in the present study coincides well with these results.

\section{Cortisol and corticosterone}

The patterns of serum CT and CCT contents observed in the present study were quite similar to that of $\mathrm{P}_{4}$. Thus, the levels of both of these hormones did not differ in 5-week-old SHR and WKY rats or in 10-weekold WKY rats, but in 10-week-old SHR rats their levels were highly elevated. The lack of differences in CT and CCT contents between juvenile SHR and WKY rats is somewhat surprising because some authors have reported that children with ADHD (Isaksson et al. 2012) as well as 6-week-old SHR rats (King et al. 2010) had lowered CT or CCT levels when compared to non-affected individuals. It is generally known that $\mathrm{CT}$ is involved in a wide range of cognitive functions (Gaysina et al. 2012) which are deficient/disturbed in ADHD children compared to normal children (executive functions: selective inhibition, working memory and plan implementation; Liu and Wang 2015). These discrepancies may be due to the differences in the age of rats and/or human children or due to the time of sample collections (Kern et al. 1996, Buckingham 2006). The elevated levels of CT and CCT in the 10-week-old SHR rats are also interesting since no significant differences in the CT levels were found between adults with ADHD and healthy controls (Corominas-Roso et al. 2015). The elevated CT content (in combination with a high content of $\mathrm{P}_{4}$ ) found in the present study was probably associated with alleviation of ADHD symptoms by this hormone, which was already discussed above (Ryan and Enns 1988). This assumption is supported by the fact that chronic adolescent CCT exposure reduces impulsive actions without any influence on their general cognitive function or attention ability in male rats (Torregrossa et al. 2012). On the other hand, excessive levels of CT and CCT in the mature SHR rats may also be associated with hypertension, as was previously reported in both rodents and humans (Yagil et al. 1996, Whitworth et al. 1998). For example, glucocorticoids may be influential in the regulation of blood pressure by stimulation of the phosphoinositide signaling system (Ohanian and Heagerty 1992). Another explanation may be autoinflammatory action, e.g. high levels of CT and CCT are connected with inflammatory response and immunosuppression (Coutinho and Chapman 2011) in which the main immunosuppressive and regulatory factors transform growth factor $\beta$ (TGF $\beta$ ). Interestingly, a decreased level of this cytokine was observed in spleens from 10-week SHR rats when compared to 5-week SHR rats and both age groups of WKY rats (unpublished data). The decreased level of TGF $\beta$ may lead to autoinflammatory action (Lifshitz and Frenkel 2013).

\section{Conclusions}

The present study, for the first time, demonstrated differences in the serum steroid hormone levels between SHR and WKY rats. Significant 
differences in the serum levels between SHR and WKY rats were mostly observed after puberty. Thus, elevated contents of $\mathrm{P}_{4}, \mathrm{CT}$ and CCT in SHR rats may be associated not only with ADHD, but also with developing hypertension, although this requires further study.

\section{Conflict of Interest}

There is no conflict of interest.

\section{Acknowledgements}

This work was supported by grant no. PL-TW II/4/2015 from the National Centre for Research and Development in Poland, Ministry of Science and Technology, Taiwan
(MOST 104-2923-B-197-001-MY3) and statutory funds of School of Medicine, University of Warmia and Mazury in Olsztyn.

\section{Author Contributions}

Anna Kozłowska conceived and designed the experiments; Pawel Wojtacha performed the ELISA procedures; Anna Kozłowska, Maciej Równiak and Małgorzata Kolenkiewicz performed the immunohistochemical procedures, Anna Kozłowska analyzed the data and wrote the paper. Maciej Równiak and Meng-Li Tsai performed paper revision.

\section{References}

AFIFY M, EL-DIEN ABD ELMAKSOUD MD, MOSA T, ELSHAERM, KOTB N: Differential effects of amitriptyline treatment on testicular and liver functions in adult male rats. New York Sci J 3: 10-18, 2010.

BENDEL P, EILAM R: Quantitation of ventricular size in normal and spontaneously hypertensive rats by magnetic resonance imaging. Brain Res 574: 224-228, 1992.

BUCKINGHAM JC: Glucocorticoids: exemplars of multi-tasking. Br J Pharmacol 147: 258-268, 2006.

BURCU M, ZITO JM, METCALFE L, UNDERWOOD H, SAFER DJ: Trends in stimulant medication use in commercially insured youths and adults, 2010-2014. JAMA Psychiatry 73: 992-993, 2016.

CASTELLANOS FX, GIEDD JN, MARSH WL, HAMBURGER SD, VAITUZIS AC, DICKSTEINDP, SARFATTISE, VAUSSYC, SNELL JW, LANGE N, KAYSEN D, KRAIN AL, RITCHIE GF, RAJAPAKSE JC, RAPOPORT JL: Quantitative brain magnetic resonance imaging in attention-deficit hyperactivity disorder. Arch Gen Psychiatry 53: 607-616, 1996.

CASTELLANOS FX, LEE PP, SHARP W, JEFFRIES NO, GREENSTEIN DK, CLASEN LS, BLUMENTHAL JD, JAMES RS, EBENS CL, WALTER JM, ZIJDENBOS A, EVANS AC, GIEDD JN, RAPOPORT JL: Developmental trajectories of brain volume abnormalities in children and adolescents with AttentionDeficit/Hyperactivity Disorder. JAMA 288: 1740-1748, 2002.

CHISTYAKOV VA, SEREZHENKOV VA, ALEXANDROVA AA, MILYUTINA NP, PROKOF'EV VN, MASHKINA EV, GUTNIKOVA LV, DEM'YANENKO SV: Effect of plastoquinone derivative 10-(6'plastoquinonyl)decyltriphenylphosphonium (SkQ1) on contents of steroid hormones and NO level in rats. Biochemistry (Mosc) 75: 1383-1387, 2010.

COROMINAS-ROSO M, PALOMAR G, FERRER R, REAL R, NOGUEIRA M, CORRALES M, CASAS M, RAMOS-QUIROGA JA: Cortisol response to stress in adults with attention deficit hyperactivity disorder. Int $J$ Neuropsychopharmacol 18: pii: pyv027, 2015.

COUTINHO AE, CHAPMAN KE: The anti-inflammatory and immunosuppressive effects of glucocorticoids, recent developments and mechanistic insights. Mol Cell Endocrinol 335: 2-13, 2011.

DEAN SL, MCCARTHY MM: Steroids, sex and the cerebellar cortex: implications for human disease. Cerebellum 7: 38-47, 2008.

DEVITO JL, GRAHAM J, SACKETT GP: Volumetric growth of the major brain divisions in fetal macaca nemestrina. J Hirnforsch 30: 479-487, 1989.

DLUZEN DE: Neuroprotective effects of estrogen upon the nigrostriatal dopaminergic system. J Neurocytol 29: 387-399, 2000.

DÖHLER KD, WUTTKE W: Changes with age in levels of serum gonadotropins, prolactin, and gonadal steroids in prepubertal male and female rats. Endocrinology 97: 898-907, 1975. 
DORN LD, KOLKO DJ, SUSMAN EJ, HUANG B, STEIN H, MUSIC E, BUKSTEIN OG: Salivary gonadal and adrenal hormone differences in boys and girls with and without disruptive behavior disorders: Contextual variants. Biol Psychol 81: 31-39, 2009.

DULCAN M: Practice parameters for the assessment and treatment of children, adolescents, and adults with attentiondeficit/hyperactivity disorder. J Am Acad Child Adolesc Psychiatry 36 (10 Suppl): 85S-121S, 1997.

GAUB M, CARLSON CL: Gender differences in ADHD: a meta-analysis and critical review. J Am Acad Child Adolesc Psychiatry 36: 1036-1045, 1997.

GAYSINA D, GARDNER MP, RICHARDS M, BEN-SHLOMO Y: Cortisol and cognitive function in midlife: the role of childhood cognition and educational attainment. Psychoneuroendocrinology 47: 189-198, 2012.

HOLMES J, HiLTON KA, PLACE M, ALlOWAY TP, ELliOTT JG, GATHERCOLE SE: Children with low working memory and children with ADHD: same or different? Front Hum Neurosci 8: 976, 2014.

HSU JW, LEE LC, CHEN RF, YEN CT, CHEN YS, TSAI ML: Striatal volume changes in a rat model of childhood attention-deficit/hyperactivity disorder. Psychiatry Res 179: 338-341, 2010.

HUISMAN HW, SCHUTTE AE, VAN ROOYEN JM, MALAN NT, MALAN L, SCHUTTE R, KRUGER A: The influence of testosterone on blood pressure and risk factors for cardiovascular disease in a black South African population. Ethn Dis 16: 693-698, 2006.

INEGBENEBOR U, EBOMOYI MI, ONYIA KA, AMADI K, AIGBIREMOLEN AE: Effect of alligator pepper (Zingiberaceae aframomum melegueta) on first trimester pregnancy in Sprague Dawley rats. Niger J Physiol Sci 24: 161-164, 2009.

ISAKSSON J: ADHD and stress. Diurnal cortisol levels, early psychosocial adversity and perceived stress. Digital Comprehensive Summaries of Uppsala Dissertations from the Faculty of Medicine 95759 pp. Uppsala: ActaUniversitatisUpsaliensis ISBN 978-91-554-8822-2, 2014.

ISAKSSON J, NILSSON KW, NYBERG F, HOGMARK A, LINDBLAD F: Cortisol levels in children with attentiondeficit/hyperactivity disorder. J Psychiatr Res 46: 1398-1405, 2012.

KALSHETTI PB, ALLURI R, MOHAN V, THAKURDESAI PA: Effects of 4-hydroxyisoleucine from fenugreek seeds on depression-like behavior in socially isolated olfactory bulbectomized rats. Pharmacogn Mag 11 (Suppl 3): S388-S396, 2015.

KAZEMI M, SAHRAEI H, AZARNIA M, DEHGHANI L, BAHADORAN H, TEKIEH E: The effect of morphine consumption on plasma corticosterone concentration and placenta development in pregnant rats. Iran J Reprod Med 9: 71-76, 2011.

KERN W, DODT C, BORN J, FEHM HL: Changes in cortisol and growth hormone secretion during nocturnal sleep in the course of aging. J Gerontol A Biol Sci Med Sci 51: M3-M9, 1996.

KING JA, BARKLEY RA, DELVILLE Y, FERRIS CF: Early androgen treatment decreases cognitive function and catecholamine innervation in an animal model of ADHD. J Psychiatry Neurosci 35: 55-58, 2010.

KLOCKE S, TAPPEHORN C, GRIESINGER G: Effects of supra-zero storage on human ovarian cortex prior to vitrification-warming. Reprod Biomed Online 29: 251-258, 2014.

LIFSHITZ V, FRENKEL D: Chapter 225-TGF- $\beta$. In: Handbook of Biologically Active Peptides (Second Edition). KASTIN AJ (ed.), Academic Press, 2013, pp 1647-1653.

LIU Y, WANG Y: Cognitive functions of children with attention deficit/hyperactivity disorder. (In Chinese) Zhonghua Yi Xue Za Zhi 82: 389-392, 2015.

LOUIS WJ, HOWES LG: Genealogy of the spontaneously hypertensive rat and Wistar-Kyoto rat strains: implications for studies of inherited hypertension. J Cardiovasc Pharmacol 16 (Suppl 7): S1-S5, 1990.

LUINE VN, JACOME LF, MACLUSKY NJ: Rapid enhancement of visual and place memory by estrogens in rats. Endocrinology 144: 2836-2844, 2003.

MARTEL MM, ROBERTS BA: Prenatal testosterone increases sensitivity to prenatal stressors in males with disruptive behavior disorders. Neurotoxicol Teratol 44: 11-17, 2014.

MCEWEN BS: Steroid hormones: effect on brain development and function. Horm Res 37 (Suppl 3): 1-10, 1992.

MORRIS JA, JORDAN CL, BREEDLOVE SM: Sexual differentiation of the vertebrate nervous system. Nat Neurosci 7: 1034-1039, 2004. 
MULLEN RJ, BUCK CR, SMITH AM: NeuN, a neuronal specific nuclear protein in vertebrates. Development 116: 201-211, 1992.

NADJAFI C: Progesterone and the nervous system. http://www.healthwatchersnews.com/2010/09/progesterone-andthe-nervous-system/, 2010.

NAGUI H: Attention Deficit Disorder (ADD) Attention Deficit Hyperactive Disorder (ADHD) Is it a product of our modern lifestyles? Am J Clin Med 6: 22-28, 2009.

OHANIAN J, HEAGERTY AM: The phosphoinositide signaling system and hypertension. Curr Opin Nephrol Hypertens 1: 73-82, 1992.

PALOMBO JD, DEMICHELE SJ, LIU JW, BISTRIAN BR, HUANG YS: Comparison of growth and fatty acid metabolism in rats fed diets containing equal levels of gamma-linolenic acid from high gamma-linolenic acid canola oil or borage oil. Lipids 35: 975-981, 2000.

PAXINOS G, WATSON C: The Rat Brain in Stereotaxic Coordinates (Fifth Edition). Elsevier Academic Press, Amsterdam, Boston, 2005, 367 p.

POPMA A, VERMEIREN R, GELUK CA, RINNE T, VAN DEN BRINK W, KNOL DL, JANSEN LM, VAN ENGELAND H, DORELEIJERS TA: Cortisol moderates the relationship between testosterone and aggression in delinquent male adolescents. Biol Psychiatry 61: 405-411, 2007.

RAMASAMY R, DADHICH P, DHINGRA A, LIPSHULTZ L: Case report: Testicular failure possibly associated with chronic use of methylphenidate. F1000Research 3: 207, 2014.

REAVEN GM, CHANG H: Relationship between blood pressure, plasma insulin and triglyceride concentration, and insulin action in spontaneous hypertensive and Wistar-Kyoto rats. Am J Hypertens 4: 34-38, 1991.

RECKELHOFF JF, ZHANG H, GRANGER JP: Testosterone exacerbates hypertension and reduces pressure-natriuresis in male spontaneously hypertensive rats. Hypertension 31: 435-439, 1998.

ROBERTS BA: "Ovarian hormones, ADHD, risk-taking, \& impulsivity". Theses and Dissertations-Psychology, 104. http://uknowledge.uky.edu/psychology_etds/104, 2016.

ROMANOS M, WEISE D, SCHLIESSER M, SCHECKLMANN M, LÖFFLER J, WARNKE A, GERLACH M, CLASSEN J, MEHLER-WEX C: Structural abnormality of the substantia nigra in children with attentiondeficit hyperactivity disorder. J Psychiatry Neurosci 35: 55-58, 2010.

RYAN EA, ENNS L: Role of gestational hormones in the induction of insulin resistance. J Clin Endocrinol Metab 67 : 341-347, 1988.

SAGVOLDEN T, JOHANSEN EB: Rat models of ADHD. Curr Top Behav Neurosci 9: 301-315, 2012.

SALERNO JA, MURPHY DG, HORWITZ B, DECARLI C, HAXBY JV, RAPOPORT SI, SCHAPIRO MB: Brain atrophy in hypertension. A volumetric magnetic resonance imaging study. Hypertension 20: 340-348, 1992.

SCHERES A, MILHAM MP, KNUTSON B, CASTELLANOS FX: Ventral striatal hyporesponsiveness during reward anticipation in attention-deficit/hyperactivity disorder. Biol Psychiatry 61: 720-724, 2007.

SCHILLING R: Alternative Treatment Of Hyperactivity (ADHD); https://www.askdrray.com/alternative-treatment-ofhyperactivity-adhd/, 2014.

SHERWIN BB: Estrogen and cognitive functioning in men with mild cognitive impairment. J Mol Neurosci 19: 219-223, 2002.

SHIVELY CA, BETHEA CL: Cognition, mood disorders, and sex hormones. ILAR J 45: 189-199, 2004.

SMITH SM. The role of the hypothalamic-pituitary-adrenal axis in neuroendocrine responses to stress. Dialogues Clin Neurosci 8: 383-395, 2006.

SONTAG TA, FUERMAIER ABM, HAUSER J, KAUNZINGER I, TUCHA O, LANGE KW: Spatial memory in Spontaneously Hypertensive Rats (SHR). Mol Cell Endocrinol 335: 2-13, 2011.

TORREGROSSA MM, XIE M, TAYLOR JR: Chronic corticosterone exposure during adolescence reduces impulsive action but increases impulsive choice and sensitivity to yohimbine in male Sprague-Dawley rats. Neuropsychopharmacology 37: 1656-1670, 2012.

VAHL TP, ULRICH-LAI YM, OSTRANDER MM, DOLGAS CM, ELFERS EE, SEELEY RJ, D'ALESSIO DA, HERMAN JP: Comparative analysis of ACTH and corticosterone sampling methods in rats. Am J Physiol Endocrinol Metab 289: E823-E828, 2005. 
WALL A, MAGNUSSON AM, MEYERSON BJ: The influences of androgen on sociosexual behavior: a comparison between the spontaneously hypertensive (SHR) and the Wistar-Kyoto rat (WKY). Physiol Behav 54: 1041-1046, 1993.

WAMBACH G, HIGGINS JR: Antihypertensive effect of progesterone in rats with mineralocorticoid-induced hypertension. Am J Physiol 236: E366-E370, 1979.

WANG LY, CHOU MC, CHOU WJ, LEE MJ, LIN PY, LEE SY, LEE YH: Does methylphenidate reduce testosterone levels in humans? a prospective study in children with attention-deficit/hyperactivity disorder. Int J Neuropsychopharmacol 20: 219-227, 2017.

WHITWORTH JA, GORDON D, ANDREWS J, SCOGGINS BA: The hypertensive effect of synthetic glucocorticoids in man: Role of sodium and volume. J Hypertens 7: 537-549, 1998.

XIAO L, BECKER JB: Quantitative microdialysis determination of extracellular striatal dopamine concentration in male and female rats: effects of estrous cycle and gonadectomy. Neurosci Lett 180: 155-158, 1994.

YAGIL Y, KOREEN R, KRAKOFF LR: Role of mineralocorticoids and glucocorticoids in blood pressure regulation in normotensive rats. Am J Physiol 251: H1354-H1360, 1996.

YAMABE N, KANG KS, LEE W, KIM SN, ZHU BT: Estriol blunts postprandial blood glucose rise in male rats through regulating intestinal glucose transporters. Am J Physiol Endocrinol Metab 308: E370-E379, 2014.

YU YZ, SHI JX: Relationship between levels of testosterone and cortisol in saliva and aggressive behaviors of adolescents. Biomed Environ Sci 22: 44-49, 2009.

ZANG YF, HE Y, ZHU CZ, CAO QJ, SUI MQ, LIANG M, TIAN LX, JIANG TZ, WANG YF: Altered baseline brain activity in children with ADHD revealed by resting-state functional MRI. Brain Dev 29: 83-91, 2007. 